\title{
Cannulation of the left common carotid artery for proximal aortic repair
}

\author{
Paul P. Urbanski, MD, Bad Neustadt, Germany
}

T he proper selection of a suitable arterial cannulation site is of great importance in aortic surgery, especially in the presence of dissection. The use of the common carotid artery for the arterial inflow offers the possibility of antegrade flow during cardiopulmonary bypass and selective cerebral perfusion during aortic arch repair.

\section{Clinical Summary}

I have used this technique in 6 patients in whom a femoral cannulation was not feasible. The heart and the proximal aorta were exposed through a median sternotomy. After mobilization of the innominate vein, the proximal segments of the arch vessels were isolated (the left common carotid artery up to a length of 3 to $4 \mathrm{~cm}$ ). In only 1 case with extensive dissection of the aorta including all supraaortic branches, the left common carotid artery was prepared via a separate incision in the neck. After heparinization, the exposed segment of the carotid artery was crossclamped; a longitudinal incision was carried out; and an 8- or $10-\mathrm{mm}$ vascular polyester graft was anastomosed to the artery with a continuous 5-0 polypropylene suture. For this purpose, a piece of a side branch of the vascular graft for later aortic replacement was used (InterGard Hemabridge with 1 side branch or InterGard Aortic Arch with 4 side branches; InterVascular, La Ciotat, France). After connection of the arterial line and cannulation of the right atrium, cardiopulmonary bypass was started with normal flow (about $2.4 \mathrm{~L} / \mathrm{min} / \mathrm{m}^{2}$ of body surface).

Because all 6 patients in whom this cannulation technique was used had aortic dissection (5 acute type A dissections and 1 persistent dissection after replacement of the ascending aorta in acute type A dissection), reconstruction was begun with the distal aortic repair. After the rectal temperature had fallen to about $26^{\circ} \mathrm{C}$, the arch arteries were clamped as far distally as possible from their origins. The left common carotid artery was clamped immediately proximal to the cannulation site, and an incomplete circulatory arrest, with selective cerebral perfusion at a flow rate of a 500 to $1000 \mathrm{~mL} / \mathrm{min}$, was instituted without crossclamping the aorta. The perfusion pressure was limited to not more than $100 \mathrm{~mm} \mathrm{Hg}$. In addition to ice packs placed around the head, thiopental and cortisone were used for pharmacological brain protection.

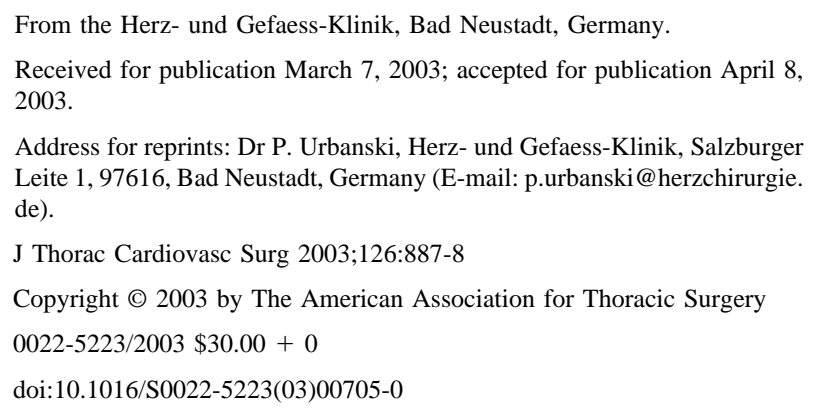

After resection of the aortic arch, an end-to-end anastomosis between the vascular graft and the descending aorta was carried out with a continuous 4-0 polypropylene suture (in 1 case using the elephant trunk technique). In 4 cases the arch arteries were transected distally to their origins and anastomosed end-to-end with the side branches of the aortic arch graft (InterGard Aortic Arch, InterVascular) with continuous 5-0 polypropylene sutures. After completion of the anastomoses to the left subclavian and the left common carotid artery, the clamps on both vessels were opened, the aortic arch graft clamped proximal to the reimplanted arteries, and cardiopulmonary bypass resumed with full flow. After anastomosing the brachiocephalic artery to the Dacron graft, the clamp was shifted proximally. In 2 patients the arch arteries were excised as a longitudinal patch and anastomosed with the Dacron graft (InterGard Hemabridge, InterVascular) using a continuous suture.

Subsequently, proximal aortic repair was carried out using our own techniques. ${ }^{1-3}$ A valve-sparing aortic root repair was performed in 3 patients, and complete replacement or re-replacement of the ascending aorta and the aortic valve with a self-assembled mechanical or biological composite graft was carried out in another 3 patients. Before termination of cardiopulmonary bypass, the arterial line was switched from the carotid artery to a side branch of the vascular graft. The Dacron graft anastomosed with the left common carotid artery was severed near the anastomosis and oversewn.

No neurological complications occurred in any of the patients. A 67-year-old schizophrenic with acute aortic dissection in association with Marfan's syndrome, however, had to be reintubated because of respiratory insufficiency and kept on long-term ventilation. This patient was transferred to a rehabilitation clinic, and the remaining patients were discharged home. At present, all patients are alive and well.

\section{Comments}

The arterial cannulation of the femoral artery generally permits rapid, simple, and low-risk installation of cardiopulmonary bypass and is widely used for surgery of the proximal aorta. There are, however, situations in which cannulation of the femoral artery is not possible or should be avoided. These include, for example, dissections extending to the iliac or femoral arteries, or severe arteriosclerosis of these vessels.

Cannulation of the right axillary artery offers antegrade flow in the aorta and cerebral perfusion during aortic arch surgery. ${ }^{4,5}$ The disadvantages of this approach lie in the necessity for an additional access and in the localization of the vessel in the neighborhood of the brachial plexus, with the potential risk of its damage during preparation. Although this cannulation method has good results in experienced hands, fatal complications have also been reported. ${ }^{6,7}$ In case of a complete arch replacement, cannulation of the axillary artery appears to prolong the operation time, in comparison with the cannulation of the carotid artery described here, and enlarges the operating field due to the additional access required. For 
complete replacement of the aortic arch, the intrathoracic isolation of the proximal segment of the arch vessels is necessary anyway. The preparation of a somewhat longer proximal segment of the common carotid artery is not time-consuming and normally does not require an additional surgical access. Even in cases where the intrathoracic cannulation is not possible, the carotid artery is better accessible than the axillary artery. Furthermore, its wall is not as delicate or as easily injured. Interruption of the arterial flow during crossclamping can be avoided by using an intraluminal shunt; however, this should only be rarely necessary, as the anastomosis with the graft usually takes only a few minutes.

Interest in the common carotid artery as an arterial cannulation site has recently increased, although in most cases it was only used for additional perfusion of the upper half of the body with simultaneous cannulation of the femoral artery. ${ }^{8}$

The cannulation technique of the left common carotid artery described offers a simple installation of cardiopulmonary bypass with antegrade flow and mostly without the necessity for a separate access in addition to the median sternotomy. Furthermore, it offers the possibility of selective cerebral perfusion during extensive operations on the aortic arch. Thanks to the absence of complete interruption of the cerebral perfusion, it minimizes the risk of air embolism and avoids the disadvantages of deep hypothermia.

\section{References}

1. Urbanski PP. Valve-preserving aortic root reconstruction (Letter). J Thorac Cardiovasc Surg. 2001;121:1220.

2. Urbanski PP. Replacement of the ascending aorta and aortic valve with a valved stentless composite graft. Ann Thorac Surg. 1999;67:1501-2.

3. Urbanski PP. Complete aortic root replacement in patients with small aortic annulus. Ann Thorac Surg. 2002;73:725-8.

4. Sabik JF, Lytle BW, McCarthy PM, Cosgrove DM. Axillary artery: an alternative site of arterial cannulation for patients with extensive aortic and peripheral vascular disease. J Thorac Cardiovasc Surg. 1995;109: 885-91.

5. Whitlark JD, Scott MG, Sutter FP. Axillary artery cannulation in acute aortic dissection. Ann Thorac Surg. 2000;69:1127-9.

6. Imanaka K, Kyo S, Tanabe H, Ohnchi H, Asano H, Yokote Y. Fatal intraoperative dissection of the innominate artery due to perfusion through the right axillary artery. J Thorac Cardiovasc Surg. 2000;120: 405-6.

7. Miyatake T, Matsui Y, Suto Y, Imamura M, Shiiya N, Murashita T, Yasuda K. A case of intraoperative aortic dissection caused by cannulation into an axillary artery. J Cardiovasc Surg (Torino). 2001;42:80911.

8. Neri E, Massetti M, Barabesi L, et al. Extrathoracic cannulation of the left common carotid artery in thoracic aorta operations through a left thoracotomy: preliminary experience in 26 patients. $J$ Thorac Cardiovasc Surg. 2002;123:901-10.

\title{
Giant atherosclerotic aneurysm of the left anterior descending artery
}

\author{
Nawwar Al Attar, FRCS, FETCS, ${ }^{a}$ Jean-Louis Sablayrolles, MD, ${ }^{\text {b }}$ and Patrick Nataf, MD, ${ }^{\text {a }}$ St Denis, France
}

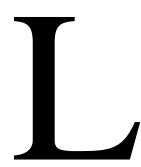

arge coronary aneurysms are mostly due to Kawasaki's disease. We report a case of giant atherosclerotic aneurysm of the left anterior descending artery (LAD) discovered incidentally in a patient with no previous coronary history. Multislice computed tomography (CT) scan of the heart gave excellent iconography, allowing accurate diagnosis and differentiating it from an aneurysm of the pulmonary artery.

\section{Case History}

A 67-year-old man of Algerian descent with no significant medical history presented with sudden onset ataxia and vertigo. The patient was overweight, afebrile, and ataxic. Blood pressure was 140/85

\footnotetext{
From the Departments of Cardiac Surgery ${ }^{\mathrm{a}}$ and Radiology, ${ }^{\mathrm{b}}$ Centre Cardiologique du Nord, St Denis, France.

Received for publication March 6, 2003; accepted for publication March 12, 2003.

Address for reprints: Patrick Nataf, MD, Department of Cardiac Surgery, Centre Cardiologique du Nord, 32-36 rue des Moulins Gémeaux, 93200, St Denis, France (E-mail: natafp@wanadoo.fr).

J Thorac Cardiovasc Surg 2003;126:888-90

Copyright (C) 2003 by The American Association for Thoracic Surgery $0022-5223 / 2003 \$ 30.00+0$

doi:10.1016/S0022-5223(03)00610-X
}

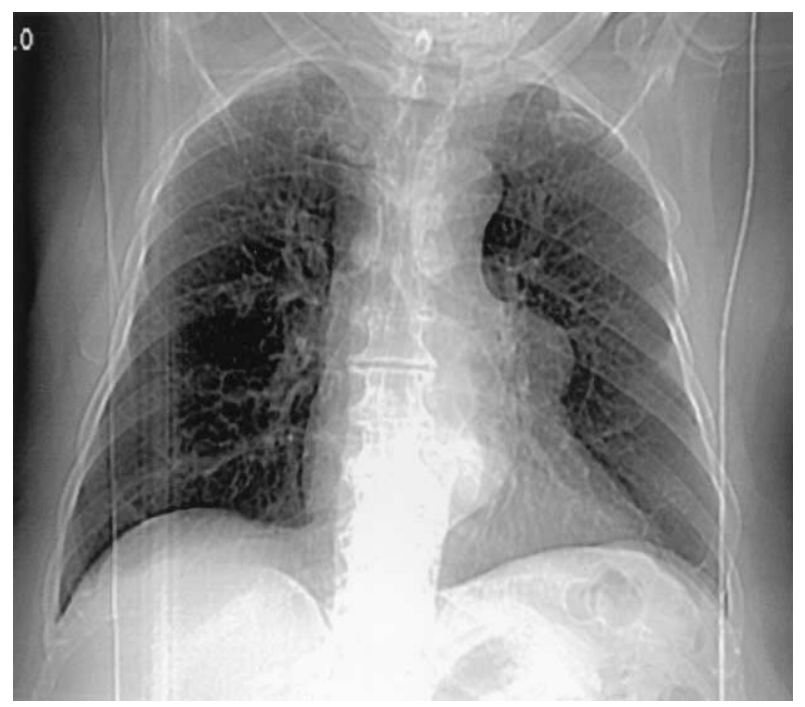

Figure 1. Chest radiograph showing left hilar mass.

$\mathrm{mm} \mathrm{Hg}$ and heart rate was regular. Brain CT scan showed left cerebellar infarction. As part of his workup, a posteroanterior chest radiograph showed a voluminous left hilar mass (Figure 1). 